\title{
John Snow, la epidemia de cólera y el nacimiento de la epidemiología moderna
}

\author{
Jaime Cerda L. y Gonzalo Valdivia C.
}

\section{John Snow, the cholera epidemic and the foundation of modern epidemiology}

John Snow (1813-1858) was a brilliant British physician. Since young he stood out for his acute observation capacity, logical thinking and perseverance, first in anesthetics and later in epidemiology. The successive outbreaks of cholera that affected London, motivated him to study this disease from a populational point of view. He related the appearance of cases to the consumption of "morbid matter", responsible for the acute diarrhea with dehydration that characterizes this disease. Bravely, Snow opposed to certain theories present at his time, sacrificing his own prestige. He was a pioneer in the use of modern epidemiological investigation methodologies such as conducting surveys and spatial epidemiology. Fairly, he is considered nowadays as father of modern epidemiology by the scientific community.

Key words: Epidemiology, Cholera, History.

Palabras claves: Epidemiología, Cólera, Historia.

\section{Introducción}

Si hubiese que enumerar algunas características de las personas que a lo largo de la historia han producido los más importantes conocimientos médicos, seguramente mencionaríamos su capacidad de observación, razonamiento lógico y una importante cuota de perseverancia. Durante su vida, John Snow encarnó fielmente todas estas virtudes. Cercano a cumplirse 150 años de su muerte, es recordado en el mundo entero por haber sido un destacado anestesiólogo, más, sobre todo, por haber sido un brillante epidemiólogo, al punto de ser considerado el padre de la epidemiología moderna. El presente artículo trae a la memoria aspectos relevantes de su vida y de su vigente legado académico.

\section{Orígenes y formación académica}

John Snow nació en la ciudad de York, Inglaterra, hacia finales del invierno de 1813.

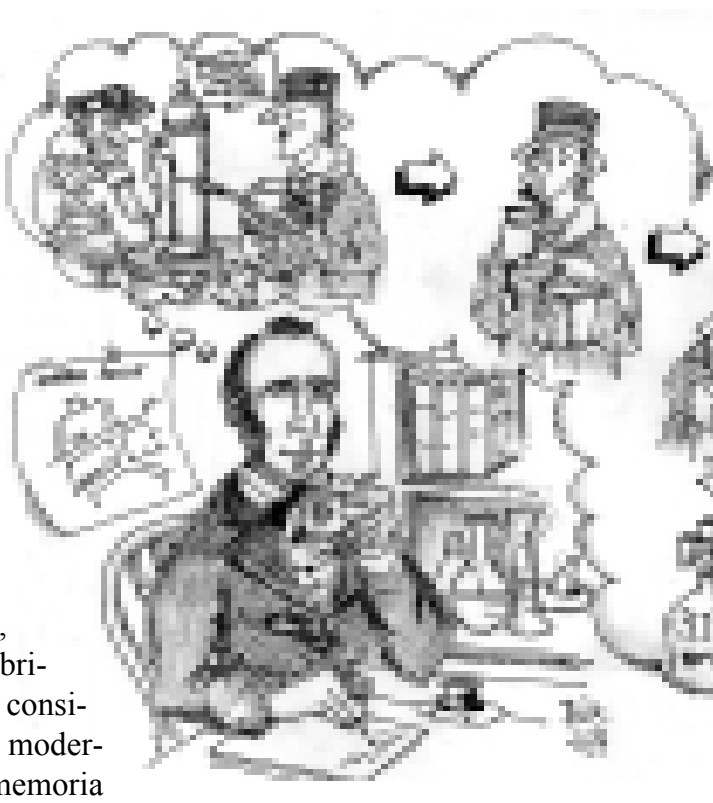

Fue el primogénito de Francis y William Snow, un matrimonio de clase media-baja. A la edad de 14 años, con la firme intención de ser médico, se convirtió en aprendiz de William Hardcastle, un cirujano-farmacéuti- co residente en Newcastle, iniciando así un estricto período de formación de cinco años.

A los 17 años, el joven Snow tuvo contacto con la primera de una serie de epidemias de una mortífera enfermedad, el cólera, durante la cual atendió a numerosos enfermos de la aldea minera de Killingsworth, experiencia que marcaría para siempre su trabajo como médico e investigador ${ }^{1,2}$.

Terminado su aprendizaje, en 1836 se trasladó a Londres y se matriculó en el Hunterian School of Medicine (f. 1769).

Durante sus años como estudiante de medicina comenzó a mostrar un agudo sentido de observación, especialmente, en las guardias que realizaba en el Westminster Hospital. Alarmado por las enfermedades que afectaban a los estudiantes que practicaban autopsias en cadáveres, Snow diseñó una serie de experimentos que demostraron la toxicidad producida por la inhalación de vapor de arsénico, metaloide utilizado para la conservación de cadáveres. 
En 1844, obtuvo su grado de Doctor en Medicina por la Universidad de Londres, estableciendo su consulta de cirujano y médico general en la céntrica zona londinense de Soho. Siempre atraído por la investigación, estudió intensamente la respiración y la asfixia, así como el comportamiento físico y químico de los gases, con especial énfasis en los gases anestésicos y su aplicación a mujeres durante el parto. Para aquel entonces, la administración de anestesia era bastante insegura debido al escaso conocimiento acerca de las propiedades farmacológicas de los gases y su errática administración. Snow diseñó un dispositivo de administración de éter y escribió una guía práctica para su uso, transformándose al poco tiempo en uno de los más prestigiosos anestesiólogos del Reino Unido. Entre sus pacientes más importantes figuró la Reina Victoria, a quien suministró analgesia con cloroformo durante el parto del príncipe Leopoldo (1853) y de la princesa Beatriz (1856). Sin embargo, no sería la anestesiología la disciplina que le daría un sitial en la historia de la medicina, sino más bien un área muy diferente, la epidemiología ${ }^{1}$.

\section{Las epidemias de cólera y el surgimiento de su hipótesis}

Durante el otoño de 1848, se produjo una segunda epidemia de cólera en Inglaterra, causando gran mortalidad. Para aquel entonces, no se conocía con certeza la etiología ni el modo de transmisión de esta enfermedad, enfrentándose dos corrientes teóricas. Por un lado estaban los "contagionistas", quienes sostenían que el cólera se adquiría por el contacto con el enfermo o con sus vestidos y pertenencias. En consecuencia, proponían medidas sanitarias drásticas como cuarentenas de buques, encierro de los enfermos en lazaretos y la quema de sus ropas y enceres. Por otro lado, estaban los que apoyaban la teoría "miasmática". Esta teoría postulaba que ciertas condiciones atmosféricas, en especial los vientos, transmitían de un lugar a otro los "miasmas": vapores tóxicos emitidos por materia en descomposición, los cuales "transportaban" de un lugar a otro el cólera. Snow no adhería a ninguna de estas teorías, en especial la teoría miasmática. Fiel conocedor del comportamiento físico y químico de los ga- ses, argumentaba su desacuerdo señalando que si la teoría miasmática fuese correcta, los pacientes deberían presentar síntomas respiratorios producto de la inhalación de los "miasmas" y no el característico síndrome diarreico agudo presente en el cólera. Preocupado por la gran mortandad a consecuencia de esta devastadora enfermedad, decidió estudiar su comportamiento epidemiológico ${ }^{2,3}$.

Basándose en el registro de las defunciones por cólera ocurridas entre 1848-49, Snow observó que los distritos de la zona sur de Londres concentraban la mayor cantidad de casos en términos absolutos y daban cuenta de la más alta tasa de mortalidad, muy superior a la del resto de la ciudad $(8,0$ y 2,4 defunciones por 1.000 habitantes, respectivamente). De igual forma, observó que los habitantes de la zona sur de Londres obtenían agua para beber río abajo del Támesis, lugar donde las aguas estaban altamente contaminadas, a diferencia de los habitantes de las demás zonas de Londres, quienes la obtenían de sectores menos contaminados aguas arriba del mismo o de sus tributarios. Con estos antecedentes en mente, Snow postuló en 1849 una innovadora hipótesis, sosteniendo que el cólera se transmitía mediante la ingestión de una "materia mórbida" invisible al ojo humano, la cual debía actuar a nivel de los intestinos, produciendo un síndrome diarreico agudo con deshidratación severa. Esta "materia mórbida" había de reproducirse y eliminarse a través de las deposiciones, las cuales, finalmente, terminaban en aguas del Támesis. La gente, al beber el agua contaminada extraída del río, ingería la "materia mórbida", cerrando así un círculo de contagio. Snow publicó su hipótesis en un artículo titulado "On the Mode of Communication of Cholera" (1849), sin em- bargo, su teoría no tuvo aceptación entre sus colegas, por el contrario, fue duramente criticado en diversas oportunidades. La comunidad médica mantenía firme sus creencias, especialmente la relacionada con la teoría miasmática ${ }^{1,4,5}$.

\section{Un experimento natural a gran escala}

En los años 1853 y 1854, Londres enfrentó una tercera epidemia de cólera. Para aquel entonces, los habitantes de ciertos distritos del sur de la ciudad extraían el agua directamente de pequeños afluentes del río Támesis o bien la obtenían a partir de numerosas bombas de agua de uso público, abastecidas por dos compañías, Southwark and Vauxhall Water Company y Lambeth Water Company. Por su parte, los desechos humanos eran vertidos en improvisadas alcantarillas o directamente al río, en una época de escasa noción de higiene ambiental. Durante la epidemia de cólera de 1848-49, ambas compañías extraían el agua de sectores contaminados del Támesis, presentando similar número de muertes los distritos abastecidos por una y otra compañía. En 1853, Lambeth Water Company había trasladado sus instalaciones río arriba, hacia un lugar de aguas impolutas, mientras que Southwark and Vauxhall Water Company mantuvo sus instalaciones en su lugar original. Al tomar conocimiento de este traslado, Snow se dio cuenta de que estaba frente a un experimento natural a través del cual podría demostrar su hipótesis. La Tabla 1 resume los resultados de su investigación (publicada en 1855), en la cual demostró que la tasa de mortalidad por cólera en hogares abastecidos por Southwark and Vauxhall Water Company era 8,5 veces mayor a la de hogares abastecidos por Lambeth Water Company (Tabla 1). Mientras realizaba su investigación, Snow

\begin{tabular}{|c|c|c|c|}
\hline Compañía de agua & Hogares & Muertes por cólera & $\begin{array}{l}\text { Muertes por } \\
10.000 \text { hogares }\end{array}$ \\
\hline$S-V$ & 40.046 & 1.263 & 315 \\
\hline L & 26.107 & 98 & 37 \\
\hline Londres (resto) & 256.423 & 1.422 & 59 \\
\hline
\end{tabular}


debió interrumpir transitoriamente sus quehaceres debido a un brote epidémico ocurrido cerca de su lugar de trabajo, el cual se transformaría en la oportunidad de agregar un sustrato empírico de su teoría ${ }^{2,5}$.

\section{La bomba de agua de Broad Street}

A principios de septiembre de 1854, un pequeño sector de Londres llamado Golden Square fue escenario de un brote epidémico de cólera de inusual intensidad, costando la vida a cerca de 500 personas en tan sólo 10 días. Como vecino del área, Snow sabía que la mayoría de los residentes del sector extraían el agua a partir de una bomba de uso público ubicada en Broad Street. Fiel a su hipótesis inicial, Snow planteó que el severo brote de cólera en Golden Square se debía a la ingestión de aguas contaminadas provenientes de esta bomba y se propuso, firmemente, demostrarlo. Para ello, tomó muestras de agua de la bomba de Broad Street y de otras cuatro bombas aledañas, compa- rando su aspecto macroscópico y microscópico. Encontró que el agua de la bomba de Broad Street tenía un aspecto más claro que las demás, sin embargo, vecinos del sector le informaron que el día anterior, sus aguas habían presentado un mal olor. Intrigado, registró los nombres y direcciones de 83 personas fallecidas en el área a causa del cólera, basándose en sus certificados de defunción y visitó algunas de sus casas, preguntando a sus moradores por la proveniencia del agua que habían bebido. Prontamente, confirmó que la mayoría de los moradores se abastecían de agua extraída de la bomba de Broad Street. Calculó la distancia entre la residencia de cada difunto y la bomba de agua más cercana, observando que en 73 de 83 casos era la bomba de Broad Street y que 61 de 83 difuntos bebían de sus aguas contaminadas en forma constante u ocasional. Entusiasmado por los hallazgos de su investigación, presentó los resultados ante la autoridad sanitaria local, quien decidió inhabilitar la bomba de Broad Street mediante la remoción de su palanca. La inhabilitación de la bomba de agua fue una medida altamente impopular entre los habitantes del sector, quienes no comprendían el sentido de la misma. Si la incidencia de casos de cólera disminuía, su teoría quedaría finalmente demostrada ${ }^{2,5}$.

$\mathrm{Su}$ rigor como investigador le hizo darse cuenta que aún faltaban algunas piezas para completar este verdadero rompecabezas epidemiológico. En su planteamiento, no hacía juicio la ausencia de muertes entre los trabajadores de Lion Brewery - una cervecería aledaña a la bomba de agua- al igual que el escaso número de defunciones ocurrido en una hospedería para gente pobre, también cercana a la fuente y en la que solamente fallecieron cinco de sus 500 huéspedes. Snow averiguó que los trabajadores de la cervecería, temerosos de beber del agua de la bomba, únicamente bebían cerveza. De igual forma, la hospedería contaba con un

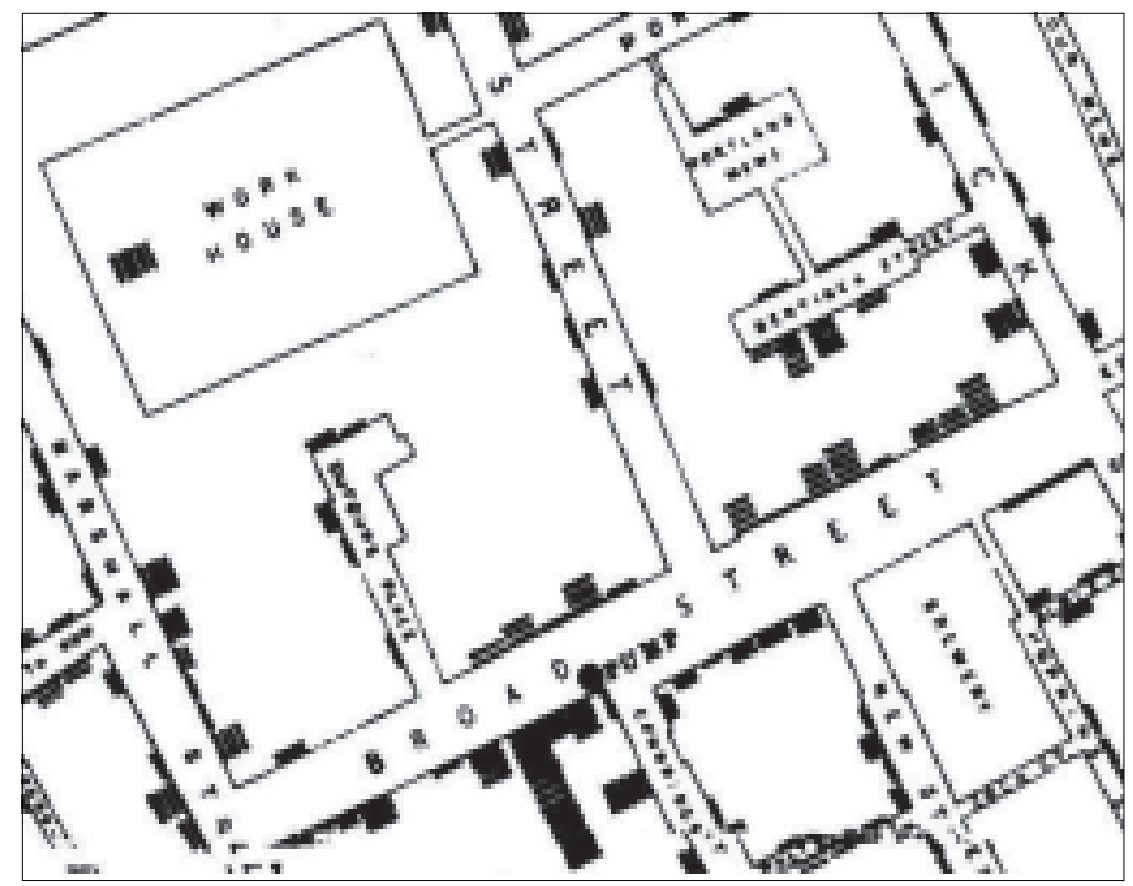

Figura 1. Mapa confeccionado por John Snow de las muertes por cólera ocurridas en el área de Broad Street ${ }^{8}$. La bomba de agua (pump) se ubica en la intersección de Broad y Cambridge Street. Las barras negras corresponden a muertes. Se observan también la cervecería (Brewery) y la hospedería (Work House).

* La fuente de este material, Wikipedia, explicita que el material es de dominio público tras la muerte de su autor.

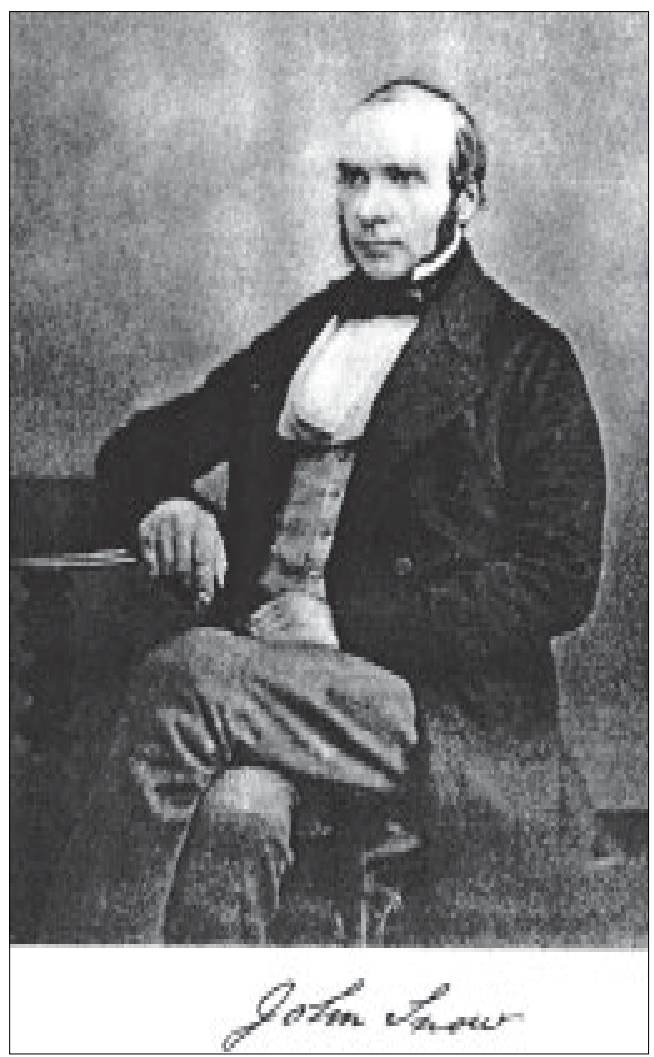

Figura 2. 
arroyo privado para el suministro de agua potable, no necesitando del agua de la bomba. Finalmente, Snow logró recabar antecedentes de algunas personas que abandonaron el sector al inicio de la epidemia y que posteriormente fallecieron en otros puntos de la ciudad libres de cólera, demostrando que habían bebido agua extraída de la bomba de Broad Street días antes de morir. Todas las piezas del rompecabezas estaban en su lugar. Para ilustrar sus hallazgos, Snow confeccionó un mapa del sector, en el cual marcó los puntos correspondientes a defunciones por cólera y las distintas bombas de agua potable existentes, demostrando gráficamente la relación espacial entre las muertes por cólera y la bomba de Broad Street (Figura 1). Finalmente, el estudio de la bomba in situ demostró que 20 pies bajo tierra, una tubería de alcantarillado pasaba a escasa distancia de la fuente de agua de la bomba, existiendo filtraciones entre ambos cursos de agua. Las denuncias de mal olor del agua emitidas por los vecinos tenían ahora una explicación lógica.

Tras la inhabilitación de la polémica bomba, se observó una reducción en la incidencia y mortalidad por cólera, sin embargo, esto no fue suficiente para controlar el brote epidémico. Lamentablemente, la incredulidad de las autoridades sanitarias -quienes apoyaban la teoría miasmática- y la presión popular fue más fuerte, habilitándose nuevamente su uso. Snow intentó hasta su muerte en 1858 convencer a la comunidad médica que el cólera se transmitía mediante la ingestión de una "materia mórbida" presente en las aguas contaminadas del río Támesis, pero sus esfuerzos fueron infructuosos. Su teoría debió esperar la cuarta epidemia de cólera de Londres, ocurrida en 1866, para ser finalmente aceptada. Al poco tiempo, experimentos realizados por Louis Pasteur demostraron que son microorganismos presentes en el ambiente (y no "miasmas") los causantes de las enfermedades transmisibles. Casi tres décadas después de la muerte de Snow,
Robert Koch aisló y cultivó el Vibrio cholerae, la "materia mórbida" a la cual recurrentemente se refería Snow, dándole total crédito a su hipótesis. Estudios contemporáneos en los que se aplicaron técnicas de regresión logística a los datos registrados en 1853 demostraron que el planteamiento de Snow siempre estuvo en lo cierto ${ }^{1,2,4-6}$.

\section{El padre de la epidemiología moderna}

En Londres, hoy en día es posible encontrar una réplica de la bomba de agua de Broad Street (actualmente Broadwick Street). Cercano a ella se ubica el John Snow $P u b$, cuyo nombre recuerda a este médico, anestesiólogo y epidemiólogo, paradojalmente abstemio. Anualmente, la John Snow Society $^{7}$ rinde un homenaje a su persona, retirando y reposicionando la palanca de la bomba de agua, como una forma de recordar los múltiples desafíos que enfrenta continuamente la Salud Pública alrededor del mundo. Sin disponer de un cuerpo de conocimientos microbiológicos y epidemiológicos como el existente hoy en día, Snow apeló a sus mejores virtudes -un agudo sentido de observación, razonamiento lógico y perseverancia- para caracterizar un problema de salud, desafiando a la comunidad médica y a la autoridad sanitaria, pensando únicamente en el bienestar de la comunidad. Con justa razón John Snow es considerado el padre de la epidemiología moderna, ejemplo del espíritu que todo médico investigador debe poseer.

\section{Resumen}

John Snow (1813-1858) fue un brillante médico inglés. Desde temprana edad destacó por su agudo sentido de observación, razonamiento lógico y perseverancia, primero en el ámbito de la anestesia y posteriormente en epidemiología. Los sucesivos brotes de cólera que afectaron a la ciudad de Londres, lo motivaron a estudiar esta enfer- medad desde un punto de vista poblacional, relacionando la incidencia de casos al consumo de aguas contaminadas por una "materia mórbida", responsable de la diarrea aguda con deshidratación que la caracteriza. En forma valerosa, Snow se opuso a las teorías vigentes de su época, sacrificando su prestigio. Fue pionero en el uso de metodologías de investigación epidemiológica moderna, por ejemplo, la implementación de encuestas y la epidemiología espacial. Con justa razón, hoy en día es considerado por la comunidad científica como el padre de la epidemiología moderna.

\section{Referencias}

1.- Ramsay M. John Snow, MD: anaesthesist to the Queen of England and pioneer epidemiologist. Proc (Bayl Univ Med Cent) 2006; 19: 24-8.

2.- Newsom S W B. Pioneers in infection control: John Snow, Henry Whitehead, the Broad Street pump, and the beginnings of geographical epidemiology. J Hosp Infect 2006; 64: 210-6.

3.- Doval H. John Show y la epidemia de cólera en Londres en 1854. Revista Argentina de Cardiología 2003; 71: 463-7.

4.- Bingham P, Verlander N Q, Cheal M J. John Snow, William Farr and the 1849 outbreak of cholera that affected London: a reworking of the data highlights the importance of the water supply. Public Health 2004; 118: 387-94.

5.- Brody H, Russell M, Vinten-Johansen P, Paneth N, Rachman S. Map-making and myth-making in Broad Street: the London cholera epidemic, 1854. Lancet 2000; 356: 64-8.

6.- Freirichs R R. John Snow. Los Angeles: UCLA Department of Epidemiology, School of Public Health. Disponible en www.ph. ucla.edu/epi/snow.html [consultado el 17/01/ 07].

7.- John Snow Society. Home page. Disponible en www.johnsnowsociety.org [consultado el 17/01/07].

8.- John Snow (physician). http://en.wikipedia. org/wiki/John_Snow_(physician) [consultado el $02 / 03 / 07]$ 